\title{
LES EXPOSANTS MORPHOLOGIQUES ET SYNTAXIQUES DE LA SYMÉTRIE EN FRANÇAIS
}

\begin{abstract}
Nkollo Mikołaj, Les exposants morphologiques et syntaxiques de la symétrie en français [Morphological and syntactic exponents of symmetry in French]. Studia Romanica Posnaniensia, Adam Mickiewicz Univcrsity Press, Poznań, vol. XXX: 2003, pp. 107-122. ISBN 83-232-1270-8. ISSN 01372475 .
\end{abstract}

The present paper deals with the exponents of symmetry in contemporary French. Apart from expressions that do not bear any markers of this type of relation, there are others where this property is closcly related to their morphemic structure. It is argued that such a morphological exponence holds when verbal stems are preceded by the prefixes entre- and co-. The forms with these markers are carefully scrutinized with regard to their combinatory and derivational features. It is claimed that the verbs with the entre-prefix are, as far as the word-formation is concerned, more regular than those with cor. If the morphemic analysis fails in explaining the semantic value of an affix, it is necessary to fall back on the description of syntagmatic properties of lexical items that incorporate it. Of special importance among syntactic exponents of symmetry is the adjunction of prepositional phrase introduced by avec. This opcration establishes uncquivocally the semantic value of verbs with coFurthermore, if such a prepositional phrase is attached to one-place predicates, it adds a new argument and becomes, jointly with the verb, the cxponent of a symmetric relation. The last syntactic exponent of symmetry dealt with here is the expression l'un l'autre. Once it is added to the verb phrase, it emphasizes the presence of such a relation in the meaning of the predicate. The paper attempts to cast a new light on the way syntactic structures of the phrases with predicative expressions that designate symmetric relations arc modified.

Les questions discutées dans l'article qui suit se situent à mi-chemin entre deux champs traditionnels de recherches linguistiques - la dérivation et la sémantique. Dès le moment où le chercheur vise à decrire la valeur d'un morphème, ces deux domaines commencent à s'enchevêtrer. C'est ainsi que le rôle sémantique d'un affixe ne peut être déterminé qu'après la segmentation de l'unité lexicale dont il fait partie. S'il contribue d'une façon non banale à actualiser la propriété sémantique dont la base dérivationnelle ne dispose pas à l'état libre, sa valeur est claire. Toutefois, la segmentation des unités s'avère souvent insuffisante. Il faut alors 
analyser les schèmes structurels (les modèles diathétiques, cf. Korytkowska 1999: 138-139) caractéristiques des unités où l'on reconnaît présent le morphème susceptible d'ajouter à la base dérivationnelle une nouvelle qualité sémantique. Il est alors possible de parler de coopération des facteurs morphologiques et syntaxiques en matière de l'actualisation d'un sens donné.

En ce qui concerne la notion de symétrie, elle peut être définie comme la propriété de certaines relations. La particularité de celles-ci est que, si elles sont vraies pour $x$ et $y$ pris dans cet ordre, elles doivent l'être également pour $y$ et $x$. Ceci veut dire que la vérité de la relation (représentable à l'aide du schème) $x R y$ implique que y $R x$ est aussi vraie pour tous les $x$ et tous les $y$ faisant partie d'un même ensemble. D'une façon formelle, la symétrie se présente donc comme dans 1:

$$
\text { 1. } \operatorname{sym}(X)=\mathrm{df}\{R: \forall x \forall y(x, y \in X \wedge x R y \rightarrow y R x)\}
$$

Pour que cette formule puisse être appliquée à l'analyse des phrases d'une langue naturelle, il faut procéder au déchiffrement des signes qui la constituent. Les symboles $x$ et $y$ représentent les variables individuelles. Autrement dit, ce sont les noms qui désignent, chacun, un des éléments d'un ensemble. Il est nécessaire de souligner qu'une variable individuelle ne réfère pas à tous les membres de cet ensemble, mais à un objet non spécifié (cf. Bańczerowski 1980: 12). Il existe deux façons d'attribuer à une variable sa dénotation. La première solution consiste à lui substituer une constante, c.à.d. à spécifier ce qui est représenté par la variable (p. ex. Max pour $x$ et Jacqueline pour $y$ ). L'autre façon de préciser sa valeur référentielle s'appuie sur l'emploi des opérateurs. Ceux-ci servent à lier les variables présentes dans la fonction soumise à la transformation (p. ex. les quantificateurs, comme dans 1 ci-dessus). Ces deux opérations permettent de transformer la fonction propositionnelle (suite de symboles contenant les variables) en une proposition au sens logique du terme (séquence de signes, douée d'une valeur de vérité, c.à.d. vraie ou fausse). Par la suite, la transformation réalisée à l'aide d'une de ces opérations sera nommée saturation. $R$, variable prédicative, peut être saturée par une expression (nom, adjectif ou verbe) précisant la nature du rapport entre $x$ et $y$ (ou entre les référents des noms introduits à leur place).

En termes de la sémantique lexicale, l'essence de la symétrie consiste dans la parité de ce qui est prédiqué de chacun des participants à la relation. Aucun d'entre eux n'est plus central (ni plus marginal) que l'autre. Ceci revient à constater que si la relation entre les individus est symétrique, chacun d'eux y est engagé de la même façon et à un même degré (Apresjan 2000: 41) ${ }^{1}$. Il en résulte que les valeurs

'Il s'ensuit que toutc démarche visant à attribuer à un syntagme nominal ou prépositionncl son rôle sémantique est, au moment où le prédicat est symétrique, vouée à l'échec. Une telle tentative a pourtant été entreprise par $\mathrm{Ch}$. Fillmore dans sa grammaire casuelle. Dans une phrase comme Monique accompagse le directeur (dont Le directeur accompagne Monique est une paraphrase fidèle), il est impossible d'attribuer à Monique le rôle d'agent (cf. Fillmore 1968: 24-25) et de confiner, en même temps, le syntagme le directeur au rôle d'objet. Le trait essenticl des arguments d'un prédicat 
logiques des phrases $2 \mathrm{a}$ et $2 \mathrm{~b}$ doivent être identiques et qu'aucune d'elles ne peut être vraie sans que l'autre ne le soit. La fausseté de la première entraîne nécessairement la fausseté de la seconde. L'inverse est aussi vrai. Les phrases où il y a un prédicat symétrique désignent un type particulier d'état de choses. Il est nécessaire que l'activité des individus dont les noms sont mis en correspondance par un élément symétrique soit concomitante. Dans les exemples qui suivent, les actions de Max et de Marie doivent avoir lieu en même temps, comme en témoigne le sentiment de bizarrerie éprouvé devant $2 \mathrm{c}$.

\section{2a. Max a épousé Marie}

2b. Marie a épousé Max

2c. ?? Max a épousé Marie en avril, alors que celle-ci l'a épousé un mois plus tard

La symétrie peut être sujette à certaines limitations. La première d'entre elles se rapporte à l'emploi des adverbes de manière. C'est ainsi que la vérité de Marie converse gaîment avec la concierge n'implique pas nécessairement celle de $L a$ concierge converse gaiment avec Marie. A son tour, la deuxième de ces phrases ne signifie pas non plus que Marie converse gaîment avec la concierge soit vraie (cf. Cresswell 1973: 140-143). En effet, bien qu'il y ait conversation entre Marie et la concierge (donc aussi entre la concierge et Marie), celle-ci peut être triste alors que Marie peut être gaie. Le second facteur non affecté par la symétrie sera discuté à la fin de l'article.

La question se pose alors de savoir si une propriété logique peut avoir les exposants dans l'inventaire des morphèmes d'une langue naturelle. A côté des prédicateurs dont la forme n'est pas révélatrice de leur aptitude à désigner une relation symétrique (cf. épouser), il y en a ceux où le sens va de pair avec la structure. Un premier pas vers la solution de cette énigme est l'analyse des verbes pronomiaux comme s'aimer, se haïr, se dévorer, se détruire, etc. Tous ces verbes sont dyadiques (Max s'aime reçoit nécessairement une lecture réfléchie) et réalisent le schème diathétique $3 \mathrm{a}$. Le symbole « $\rightarrow$ indique la concaténation. Les positions des arguments $\mathrm{N}_{1}$ et $\mathrm{N}_{2}$ sont, bien entendu, interchangeables (ce qui est représenté par les parenthèses). Les noms des actants peuvent être également sujets à lunification réalisée au moyen du pronom personnel ils ou elles (cf. Lewicka et Bogacki 1983: 9).

symétrique est leur égalité mutuelle. Le chercheur qui s'obstine à caractériser le rôle de Monique et celui de le directeur dans l'exemple ci-dessus se trouve donc devant le choix: (i) soit assigner un double statut sémantique à chacun des syntagmes (ce qui est une complication peu désirable el s'oppose à la règle de Fillmore «une fonction sémantique par $\mathrm{SN}$ »); (ii) soit introduire une règle assurant l'identité des rôles qu'assument les arguments des prédicats comme épouser ou accompagner. Cette deuxième solution va à l'encontre d'un autre principe prôné par Fillmore, à savoir «une fonction sémantique par phrase». 
3a. $\mathrm{N}_{1}\left(\mathrm{~N}_{2}\right)-$ et $-\mathrm{N}_{2}\left(\mathrm{~N}_{1}\right)-$ se $\mathrm{V}$

3b. ils $\left(=N_{1}\right.$ et $\left.N_{2}\right)-$ se $V$

Cependant, l'actualisation de cette structure conduit à la production des phrases ambiguës. En effet, dans Marie et Pierre s'aiment, la valeur sémantique du pronom personnel est loin d'être claire. Cette phrase peut signifier tantôt que Marie aime elle-même et que Pierre aime lui-même (le pronom est donc réfléchi et réalise le schème $x R x \wedge y R y$ ), tantôt que Pierre aime Marie et que Marie aime Pierre (le pronom est «réciproque» et actualise la relation $x R y \rightarrow y R x$ ). Il est possible de lever cette ambiguïté, ceci à l'aide d'un morphème synsémantique. Il est question ici du préfixe entre-. A l'opposé de Marie et Pierre s'aiment, la phrase Marie et Pierre s'entr'aiment ne recèle plus d'ambiguité.

Il convient, au moment où l'on aborde la description de la valeur sémantique des verbes français à préfixe entre-, d'esquisser quelques problèmes relatifs à leur niveau dérivationnel. Parmi 37 verbes qui contiennent ce formant, cités par Le Nouveau Bescherelle, la plupart désignent une relation symétrique ${ }^{2}$. En outre, la base dérivationnelle qui, accompagnée du préfixe en question, forme un verbe nouveau, existe indépendamment dans le système lexical français. Or, la motivation de ces verbes est, en ce qui concerne le niveau formel, pleine. Autrement dit, les verbes à préfixe entre- actualisant les relations symétriques sont fondés sur le parcours dérivationnel suivant: $\operatorname{Pref}_{1} \mathrm{BD}_{1}, \operatorname{Pref}_{1} \mathrm{BD}_{2}, \operatorname{Pref}_{1} \mathrm{BD}_{3}, \operatorname{Pref}_{1} \mathrm{BD}_{\mathfrak{n}}$, etc. (cf. Giermak-Zielińska 1979: 83). Ceci veut dire qu'il existe une association constante entre le préfixe et une série de bases dérivationnelles. Celles-ci fonctionnent, toutes, d'une façon autonome dans le lexique. Après l'adjonction du formant entre-, leur sens acquiert un caractère réciproque. Désormais, le verbe désigne une relation symétrique. Avant l'adjonction de entre-, une équivoque risquait de se former (à l'opposé de Pierre et Paul s'entraccusent, la phrase Pierre et Paul s'accusent signifie plutôt que chacun d'eux se reconnaît coupable). D'autres caractéristiques de ces verbes, c.à.d. le sens, la valence et la valeur casuelle des syntagmes qui leur sont obligatoirement attachés, restent intacts. L'unique exception est le verbe s'entretenir dont le sens s'écarte de celui qui caractérise sa base dérivationnelle - tenir. La

"Le prófixe en question connaît également les emplois au sens «incomplètement, partiellement», comme ceci a lieu dans: entrebâiller, entrouvrir. Ces verbes ne renvoient pas à une relation symétrique. En outre, il existe des verbes où il n'y a aucun rapport sémantique entre le formant el la base dérivationnelle (entraîner à côté de traîner, entreprendre à côtć de prendre, entrevoûter à côté de voûte, etc.). Le préfixe entre- (qui impose une valeur symétrique à l'élément qu'il modifie) reste généralement disponible en français contemporain. Les formes verbales cntrées depuis longtemps dans la langue sont écrites en un scul mot (s'entremêler). Les formations occasionnelles requièrent un trait d'union. L'emploi du préfixe en question était encore plus répandu qu'aujourd'hui en ancien français, cf. «Uns vavasors andemantiers antra leanz, qui mout lor nut, qui monseignor Gauvain conut, si les trova antrebeisant et mout grant joie antrefeisant) (Perccval, 5766-5770). Il est curieux de noter que les verbes à préfixe entre- ne requéraient pas la présence du pronom. 
motivation de cette classe de composés est donc à peu près pleine aussi au niveau sémantique ${ }^{3}$.

La structure syntaxique que revêtent les phrases avec un des verbes symétriques à préfixe entre- est intimement liée au niveau dérivationnel de ceux-ci (les bases de tous les verbes cités par Le nouveau Bescherelle sont soit dyadiques dévorer, nuire, tuer - soit triadiques - accorder, accuser; on peut donc légitimement s'attendre à ce qu'il en soit de même avec les dérivés). Toutefois, la quotepart des propriétés logiques des éléments prédicatifs semble encore plus importante lors de la description du comportement syntaxique des phrases où ils figurent (cf. Korytkowska 1999: 138). Pour qu'il y ait symétrie, il doit y avoir au moins deux participants à la relation. C'est pour cette raison que les verbes monadiques (se réveiller, bâiller, dormir, etc.) sont exclus du présent champ d'investigation. La nature symétrique de la relation désignée par les verbes à préfixe entre- entraîne l'interchangeabilité des positions d'arguments. Les configurations possibles sont indiquées dans le schéma ci-dessous.
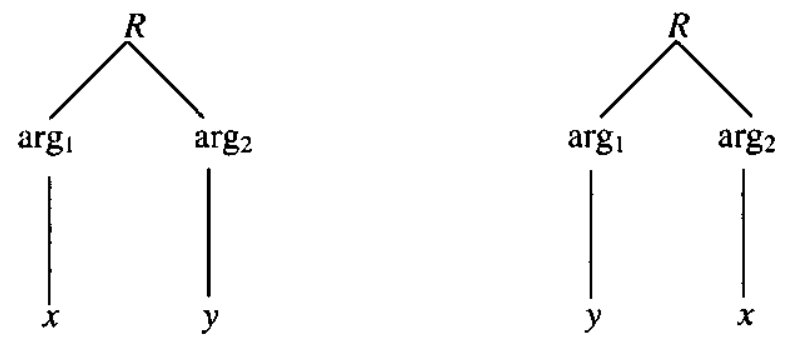

${ }^{3}$ La question qui mérite d'être éclairée est de savoir quelles contraintes sont imposées aux bascs dérivationnelles combinables avec le préfixe entre-. Comme il a déjà étế indiqué, le verbe ne peut pas être monadique, car une relation symétrique nécessite la présence de deux actants (ou plus). Cependant, il existe des verbes dyadiques et triadiques auxquels il est impossible de rattacher le formant entre-. La première restriction est de nature sćmantico-logique. Il est interdit de joindre le formant en question à un verbe qui désigne déjà une relation symétrique (*s'entre-coïncider, *'s'entre-coopérer, *s'entreépouser, *s'entre-accompagner, etc.). L'autre limitation concerne les restrictions sélectives imposées par l'élément prédicatif à ses arguments. Ceux-ci doivent ĉtre de nature homogène. La raison profonde de cet état de choses est expliquée dans la définition l ci-dessus - la symétrie n'a lieu qu'entre deux (ou plusieurs) éléments appartenant à un même ensemble. Là où le verbe postulc p. ex. un sujet humain et un objet non-animé, il est facile de prouver que les objets ne font pas partie d'un même ensemble. Ceci explique les difficultés éprouvées devant s'entre-stocker (< stocker une marchandise), etc. Enfin, une dernière limitation sc rapporte au niveau morphotactique de la langue. Cette contrainte suggère qu'il existe des entraves phonétiques qui s'opposent à la combinaison d'un verbe donné et du préfixe entre(cf. la discussion autour des contraintes de ce type chez Aronoff 1976: 79-81; voir aussi Nagórko 2001: 183-186 et son concept de "slowotwórstwo morfonologiczne»). C"est ainsi qu'une cacophonie risque de se produire au moment ou l'on conglomère en une seule unité un verbe commençant par entre. et le formant entre- (Ces deux fabricants s'entre-entreposent des marchandises par courtoisie). Cette règle s'applique chaque fois que la base dérivationnelle reproduit les éléments consonantiques du préfixe (p. ex. Les deux alchimistes s'entre-transforment en monstres). 
Puisque les relations symétriques engagent deux actants, le nom de chacun d'eux doit être spécifié dans la phrase avec les verbes à préfixe entre-. Si cette condition n'est pas respectée, la construction est sémantiquement incomplète et syntaxiquement incorrecte (*Pierre s'entre-hait, *Je vis grâce à l'entraide de Max). On voit donc, une nouvelle fois, que les propriétés sémantiques (en l'occurrence, la parité de ce qui est prédiqué de chacun des individus) sont révélatrices de la forme que revêtent les constructions avec ce type de prédicats (il est évidemment possible de saturer les positions d'arguments par des expressions référentiellement vides quelque chose ou quelqu'un). Ces proprictés expliquent également la nature et l'origine des réaménagements qui s'observent là où l'on a affaire aux phrases avec un prédicat symétrique. La structure actancielle des verbes à préfixe entre-est donc analogue à celle qui a été indiquée à l'occasion de la description des verbes pronominaux correspondants (cf. $3 \mathrm{a}$ et $3 \mathrm{~b}$ ci-dessus). L'argument qui, dans une première structure, occupe la première position, peut être repoussé à la seconde position dans la deuxième structure et inversement. Les deux termes du syntagme sujet coordonnés par et peuvent être abrégés à la forme d'un nom (ou d'un pronom personnel) au pluriel. Une position syntaxique correspond dans ce cas-là à plusieurs arguments. Cf. 4.

\section{4a. Paul et Guillaume s'entraccusent d'avoir commis ce vol 4b. Guillaume et Paul s'entraccusent d'avoir commis ce vol $4 \mathrm{c}$. Ils s'entraccusent d'avoir commis ce vol $\mathrm{t}^{4}$}

Comme il a été dit, les verbes précédés du préfixe entre- existent d'une façon autonome dans le lexique. L'adjonction de ce formant leur permet d'acquérir un caractère symétrique dont ils sont dépourvus à l'état libre. Il en est un peu autrement avec les verbes qui contiennent le préfixe $c o$-. Cette classe s'avère plus hétérogène à tous les niveaux d'analyse. Il convient de commencer la description par élucider quelques problèmes relatifs à leur dérivation. A l'opposé des verbes à entre- où la

${ }^{4}$ La question se pose de savoir quelles sont les propriétés qui distinguent le formant entre- de son pendant scientifique inter-. Bien que ces deux préfixes soient généralcment présentés enscmble dans des ouvrages traditionnels et didactiques consacrés à la dérivation en français (du point de vue étymologique, ils forment un doublet), certaines différences les séparent. En ce qui concerne les verbcs symétriques, on ne retrouve inter- que dans un groupc très rcstreint de ceux-ci (interagir, interdépendre, s'interpénétrer, ctc.). Cet affixe, tout en étant moins fréquent que entre-, modifie plutôt le signifié des noms (intersection, intercommunion, interversion, interchangeabilité, interconnexion, etc.). Dans cette fonction aussi, sa productivité est peu élevée. En outre, inter- se joint de préférence aux bases directement empruntées au latin. Maintes fois, cet élément figure dans des verbes venus tels quels de cette langue (interroger, interrompre, intercepter, etc.). A l'opposé de leurs homologues symétriques à préfixe entre- quj sont à peu près toujours motivés formellement et sćmantiquement (exception faite de s'entretenir), les verbes avec inter- ont généralement soit un sens local («au milieu de» - interpoler, interfolier, interclasser, etc.), soit leur sémantisme n'a rien à voir avec celui que vćhiculent leurs parties constitutives (cf. les mots venus directement du latin énumérés ci-dessus). Dans ce cas-là, il n'y a qu'une motivation formelle. 
base dérivationnelle faisait toujours partie du système lexical français, les verbes qui commencent par co-sont moins réguliers. En témoigne l'observation des schèmes dérivationnels qu'ils réalisent. Les associations suivantes peuvent y coexister:

(i) Pref, $\mathrm{BD}_{1}$, Pref $_{1} \mathrm{BD}_{2}, \operatorname{Pref}_{1} \mathrm{BD}_{3}$, Pref $_{1} \mathrm{BD}_{\mathrm{n}}$, etc.: coordonner (< ordonner), concélébrer (< célébrer), cohabiter (< habiter), coexister (< exister), conjoindre (< joindre; le préfixe n'entraîne ici aucune modification sémantique). Cependant, le lexique français abonde en formations composées auxquelles il est parfaitement possible de trouver une base dérivationnelle autonome, mais qui sont dépourvues de caractère symétrique (consentir, connoter, contenir, comprendre, compromettre, etc.). Elles n'exhibent alors aucun lien sémantique avec le lexème formellement motivant.

(ii) Pref, $\mathrm{BD}_{1}, \operatorname{Pref}_{2} \mathrm{BD}_{1}, \operatorname{Pref}_{3} \mathrm{BD}_{1}, \operatorname{Pref}_{\mathrm{n}} \mathrm{BD}_{1}$, etc.: conglomérer (à côté de agglomérer, à l'exclusion de glomérer), confronter (à côté de affronter, à l'exclusion de fronter), conglutiner (à côté de agglutiner, à l'exclusion de glutiner), converger (à côté de diverger, à l'exclusion de verger), etc. Cf. la notion de «podstawy związane) (bases dérivationnelles non libres) chez Grzegorczykowa et Puzynina (1999: 365). L'existence d'un tel rapport s'avère parfois trompeuse (le sens de l'unité supposée assumer la fonction de base dérivationnelle varie d'un composé à l'autre), comme ceci a lieu avec conspirer (à côté de inspirer, aspirer, à l'exclusion de spirer), conjuguer (à côté de subjuguer, en l'absence de juguer). Loin d'être une caractéristique exclusive des verbes symétriques, ce type de motivation s'observe également là où il en vient à la description des unités dont le sens n'exhibe pas cette propriété. Cf. consulter (à côté insulter, à l'exclusion de sulter), congréer (à côté de maugréer, agréer, à l'exclusion de gréer), etc.

(iii) Pref $\mathrm{BD}_{1}$ et la base dérivationnelle $\left(\mathrm{BD}_{1}\right)$ où elle fonctionne d'une façon autonome dans le lexique et appartient, en même temps, à une autre partie du discours. C'est le cas de: coïncider (à côté de incident), commercer (à côté de commerce; lat. mers, -cis «marchandise»), commérer (à côté de commère), copiner (à côté de copain), etc. Cf. la discussion autour des problèmes relatifs à ce type de motivation chez Matthews (1991: 63-69). D'un autre côté, on observe aussi dans la classe de verbes non symétriques à formant co- l'existence de ce type de rapport. II est alors question des verbes parasynthétiques comme contrister (triste), de même que de consigner (signe), commémorer (mémoire), concentrer (centre), etc. On voit donc qu'à chaque règle dérivationnelle dans ce groupe correspond une série de contre-exemples annihilant toute tentative de systématisation.

On doit noter en même temps que la symétrie de la relation inscrite dans le signifié du verbe ne suffit pas à garantir l'existence d'une base dérivationnelle précise. Ceci veut dire qu'il existe des unités qui ne peuvent pas, sur le plan synchronique, être rattachées à quoi que ce soit d'autre dans le lexique. Tel est le statut de combiner, se coaliser (coalition n'est ni formellement ni sémantiquement primaire), collationner, concilier, etc. Parmi les verbes non symétriques qui incorporent le préfixe en question, le manque de motivation s'observe aussi. Les 
formes constiper, contaminer, convoiter, contempler, etc. en disent long. Il n'existe alors, au niveau dérivationnel, aucun trait distinctif permettant de nettement opposer la classe de verbes symétriques à ceux où cette caractéristique est absente.

Les verbes français à préfixe $c o$ - sont très nombreux, mais il n'y a parmi eux que trois dizaines de ceux qui sont symétriques. En outre, la base dérivationnelle y fait souvent défaut (ce qui est vrai aussi des verbes non-symétriques où l'on reconnaît la présence de ce morphème). Il paraît donc pertinent de se poser la question si le préfixe co-faisant partie des formes verbales en question est réellement un formant et si l'étude de la structure de ces verbes relève de la morphologie dérivationnelle. En effet, quelle valeur peut-on attribuer à co- dans construire (nonsym.) ou collationner (sym.) étant donné que les prétendues bases dérivationnelles -struire et -lationner n'existent pas dans le lexique? Les arguments historiques et comparatives (cf. les mots structura et struere en latin et le verbe russe строить) sont peu maniables'.

Puisque les critères morphologiques s'avèrent insuffisants, c.à.d. il est impossible, à partir de leur connaissance, de déterminer d'une façon univoque si la présence de co-est un exposant de la symétrie, le problème doit être tranché par l'étude des structures syntaxiques qu'imposent aux phrases les expressions incorporant ce formant. La nature sémantique des verbes avec ce préfixe se reconnaît à travers leur combinabilité. Dès le moment où une expression de ce groupe postule dans son entourage la présence obligatoire d'un syntagme prépositionnel introduit par avec, elle est indéniablement symétrique. Quant à la valeur casuelle de ce syntagme, on y reconnaît un comitatif. C'est le cas qui indique les objets accompagnants (cf. Kempf 1978: 98-99). L'accompagnement étant une relation symétrique, on ne s'étonne pas devant la combinabilité obligatoire d'un prédicat symétrique et d'un syntagme comitatif. C'est ainsi que le verbe coüncider requiert la présence de deux arguments. Sauf celui qui est exprimé par le terme qui assume la fonction sujet (le nominatif), il doit être accompagné d'un complément. Le référent de celui-ci indique un

${ }^{5} 11$ y en a pourtant un qui doit ctre mentionne et qui incline à localiser la description de ces verbes dans le cadre de la composition. Jl est de notoriété parmi les romanisants de reconnaître quc, dans les mots latins, les groupes consonantiques disjoints (formés d'unc consonne terminant la syllabe précédente ct d'une consonne initiale de la syllabc suivante) ont été sujets à des modifications phonétiques parfois sensibles. C'est, entre autres, le cas du groupe [ns] comme dans les mots maison < masione < mansione ou coûtume < costume < consetumine < consuetudinem (cl. Fouchć 1961: 8108It). Généralement, c'était le [n] qui est tombé, et ceci déjà à l'époque latine. Lc groupe [ns| s'est cependant conservé dans un certain nombre de mots (génćralement sentis comme savants). [l est question ici surtout des composés à premier élément in-ou con-. A cause du sentiment de composition ct aussi par analogic avec d'autres mots où in- et con-étaient suivis d'unc consonnc autre que [s], ils ont conservé la nasalc: insimul > ensemble, consenvare > conserver, consentire > consentir, etc. A côté d'eux, il existait d'ailleurs des formes simul, servare, sentire, etc. En dehors de ces cas, on a affairc d des formes composées ou non dont le caractère plus ou moins savant se révèle par la comparaison, la sémantique ou la date tardive d'attestation: consolation, consomation, consanguin. consul, consignation, consort, consulter, etc. 
événement concomitant (accompagnant celui qui est désigné par le premier argument). Désormais, il devient clair pourquoi il est possible d'intervertir les positions d'arguments dans Ton départ a coüncidé avec ma venue. En effet, la parité de ce qui est prédiqué de l'un et de l'autre événement autorise les usagers à transformer la phrase ci-dessus en Ma venue a cö̈ncidé avec ton départ. Cette mutation n'affecte aucunement la valeur logique (la vérité ou la fausseté) de ces deux expressions. Ce critère permet de déceler, dans la classe de verbes avec le préfixe $\mathrm{Co}_{-}$, les éléments suivants: se coaliser, coexister, cohabiter, coincider, collaborer, collationner, combiner, commercer, commérer, communiquer, comparer, concélébrer, se concerter, concilier, concorder, conférer, confondre, confronter, conglomérer, conglutiner, conjoindre, conjuguer, se conjurer, connecter, consoner, conspirer, contraster, convenir, converger, converser, coopérer, coordonner, copiner, correspondre («s'écrire mutuellement») ${ }^{6}$.

Comme les relations symétriques présupposent la participation de deux actants, il n'est généralement pas possible d'omettre le deuxième argument. Une telle omission conduit soit à une construction syntaxiquement incorrecte (*Il collabore, à l'opposé de la proposition habituelle-potentielle Il collabore volontiers, Il se marie sur un coup de tête, cf. Karolak 1999: 131; Lamiroy 1993: 252-253), soit à une phrase qui requiert la coopération du contexte situationnel pour être comprise (Ce dimanche, l'évêque va concélébrer la messe dans notre paroisse, avec qui?). Certaines restructurations à l'intérieur des phrases avec un des verbes de cette série peuvent être pratiquées. Il est possible de repousser le syntagme-objet devant le verbe et de le mettre en correspondance avec le premier argument au moyen de $\boldsymbol{e t}$. Si les deux arguments sont de nature homogène, il est possible de les comprimer à la forme du pronom personnel pluriel. Toutes ces modifications sont admissibles

${ }^{6}$ Le verbe concourir forme un problème à part. Son modèle syntagmatique (acception: «tendre cnsemble vers un même but") n'admet généralement pas la présence d'un syntagme comitatif introđuit par avec. Cependant, ce verbe implique la participation de deux actants (ce qui permet, en définitive, de le qualificr de symétrique), comme on s'en aperçoit dans les cxemples suivants: Tous les détails' de composition concourent à l'harmonie générale du tableau, Les danses folkloriques ont concouru à donner à la fête tout son éclat, etc. On a affaire ici à la réduction (pratiquée souvent si les actants sont deux spécimens appartenant à une seule classe d'objets) des noms des actants à une forme plurielle. Ce détail est nécessaire à la bonne formation grammaticale de la phrase, car dans Une danse folklorique a concouru à donner à la fête tout son éclat, une position d'argument fait défaut (a concouru avec quoi?). La présence obligatoire d'un syntagme comitatif auprès des verbes à préfixe $c o$ - suffit à leur conférer un caractère symétrique, mais elle n'est pas une condition nécessaire. Concourir n'est pas l'unique exception. Il en est de même avec le verbe correspondre ("être conforme à, être identique à»). Celui-ci désigne incontestablement une relation symétrique. On peut s'en convaincre grâce aux exemples: $\mathrm{Ce}$ mot de notre langue correspond au terme anglais que tu as mentionné, Le terme anglais que tu as mentionné correspond à ce mot de notre langue, le terme anglais que tu as mentionné et ce mot de notre langue se correspondent. L'identité des conditions de vérité de ces trois phrases est évidente. La formule $x R y \rightarrow y R x$ s'applique donc ici. L'unique facteur qui distingue correspondre des autres verbes est son comportement syntagmatique: il exige l'emploi de $a$ au lieu de avec. 
à condition de mentionner l'un et l'autre participant à la relation désignée par le verbe. Cf. les modèles syntaxiques dans 5 et les exemples dans 6. Ils s'appliquent aux prédicats dyadiques?

5a. $N_{1}\left(N_{2}\right)$ - verbe - avec $-N_{2}\left(N_{1}\right)$

$5 b . N_{1}\left(N_{2}\right)$ et $N_{2}\left(N_{1}\right)-$ verbe

5c. Pronom personnel (au pluriel) - verbe

\section{6a. Pauline cohabite avec les Dupont}

6b. Les Dupont cohabitent avec Pauline

6c. Pauline et les Dupont cohabitent

6d. Les Dupont et Pauline cohabitent

6e. Ils cohabitent

Il est intéressant, quand on aborde le problème d'un point de vue historique, de s'interroger sur les causes de la divergence formelle du préfixe co- et du morphème avec en français. D'autres langues romanes s'avèrent plus conservatrices et continuent à exhiber le parallélisme morphologique entre co- et la préposition quj succède aux verbes symétriques de ce type (cf. l'italien: La data del tuo arrivo coincide con quella della mia partenza, Le tue aspirazioni convergono con le mie, Il testimone ha collaborato con la giustizia, etc.; il se crée ici une redondance dans la transmission des informations lexicales et sémantiques). Or, le latin connaissait l'analogie du formant $c o$ - et de la préposition cum. L'écroulement du système phonologique de cette langue et la disparition des oppositions dues à la durée vocalique (le nominatif pirată était distinct de l'ablatif piratā et ne se confondait pas avec lui) ont débouché sur l'ouverture des $u$ brefs latins en $o$. La préposition cum où le $u$ était bref a abouti à la forme com. A ce moment-là, une homonymie gênante entre com issu de cum et com issu de quomo (lat. class. quomodo) risquait de se former (cf. «Li Tens qui ne puet sejorner Ainz vait toz jorz senz retorner, Con l'eve qui s'avale toute», Roman de la Rose, 373-375). La situation était d'autant plus compliquée que l'ancien français était sujet à des variations dialectales multiples et que les copistes

${ }^{7}$ Il convient de se demander sur ce qu'il en est avec la symétrie et quels arguments des prédicats triadiques peuvent figurer dans la formulc $x R y \rightarrow y R x$. L'cxcmple des verba iungandi (combiner, concerter, conglomérer, conglutiner, conjoindre, conjuguer, connecter pour ne citer que ceux qui commencent par co-) est particulièrement éclairant à cet égard. Puisqu'ils sont, tous, cn même temps triadiques ct causatifs, il est facile de reconstituer leur schème diathétique. Le premier argument doit renvoyer à l'auteur de la causation, les deux autres participent à une relation symétrique qui consiste dans une localisation commune de dcux objets ( $x$ se trouve avec $y$ ct $y$ se trouve avec $x$ ). D'où l'interchangeabilité de Pour y aboutir, Max a dû conjuguer son intelligence et sa ténacité et Pour y aboutir, Max a dû conjuguer sa ténacité et son intelligence. La possibilité de permuter le deuxic̀me et le troisième arguments des verba iungandi résulte du caractère symétrique de la relation «être ensemble» qui fait partie du signifié de concerter, conglomérer, conglutiner, etc., ainsi que de leurs correspondants sans préfixe co-(accoupler, appareiller, joindre, unir, etc.). 
se permettaient des licences d'ortographe (à côté de com, on notait la forme cum "Ne sai vus dire cum ler ledece est grande», Vie de Saint Alexis, 610; même avec les emplois temporels, "Cum veit le lit, esguardat la pucela», Vie de Saint Alexis, 56; cf. Imbs 1956: 143-144). Une phrase comme Il se querelle *com sa femme, pourrait signifier tantôt $l l$ se querelle avec sa femme, tantôt $l l$ se querelle comme sa femme (c.à.d. il est aussi querelleur qu'elle). C'est pour cette raison que la forme avec, issue de apud hoque, a évincé le latin cum $^{8}$.

Le caractère prédicatif et la symétrie ne sont, bien entendu, pas l'apanage des verbes. Les notions abstraites (ies noms de phénomènes, qualités, idées, procès, etc.) sont susceptibles d'être exprimées également à l'aide des substantifs. Ceux-ci ont, à un même titre que les verbes, des caractéristiques diathétiques précises. Afin d'acquérir une valeur référentielle pleine, un nom comme indignation exige que toutes les positions d'arguments qu'il ouvre soient saturées (cf. Karolak 1990: 284). Autrement dit, pour rendre sémantiquement complets les syntagmes nominaux dont les substantifs abstraits comme indignation sont les déterminés absolus, il est nécessaire d'expliciter l'identité des actants. C'est ainsi qu'on rapporte le nom indignation à celui qui éprouve ce sentiment, à celui qui en est la cause et à son acte (p. ex. $L$ 'indignation de Max contre ses parents en rapport avec leur visite inattendue $\grave{a}$ Bucarest). La situation est analogue avec certains noms à préfixe $c 0-$. Ce formant est responsable de l'introduction d'une nouvelle information sémantique et suggère qu'il existe plusieurs individus dont l'identité est précisée par la base dérivationnelle. Le syntagme nominal dans Le congénère (compagnon, copain, consort, confrère, etc.) est vorace est référentiellement incomplet. Il est nécessaire, au moment où l'on cherche à le transformer en une description définie (référentiellement univoque), d'indiquer de qui l'individu en question est congénère, compagnon, etc. Ce n'est donc qu'à condition de saturer toutes les positions d'arguments que se constate la symétrie des noms prédicatifs à co-. Or, la syntaxe vient,

${ }^{8}$ Brøndal (1950: 105-107) souscrit, non sans certaines réserves, à une hypothèse tout à fait différente en matière de l'origine de la substitution de apud hoque à cum à l'époque de la formation du français. Cctte perturbation a, selon lui, un caractère local et culturel et s'explique par une influence du gaulois. L'argumentation mise en avant à l'appui de cette idée est cependant peu claire. Puisque l'idiome gaulois est méconnu de nos jours, Brøndal s'adresse à l'irlandais (le plus proche dans le temps du gaulois). Selon lui, les formes amal et con qui correspondent dans cette languc à avec du françajs contemporain et à cum latin ont été délaissées: amal est conjonction («de même que») et con sert de préfixe. Les deux correspondants irlandais de apud latin et de chez français: la et oc leur sont préférés. Ces deux formes servent, l'une et l'autre, à exprimer l'objet ou l'individu accompagnant. L'autre fait évoqué par Brøndal provient de l'obscrvation de l'anglais parlé en Irlande où il est, selon lui, courant de dire $1 t$ was done with him pour It was done by him. D'après le chercheur danois, de même que dans ces exemples l'anglais with - contre son usage de base - rend l'irlandais le, de même on peut supposer que lc gallo-roman apud - pour le lat. cum - a rendu cette même préposition sous sa forme gauloise. En effet, on observe dans le latin parlé en Gaule les phrases "vixit apud eum annos XXIII" (au lieu de "cum e(o» - inscription tombalc à propos d'une femme el de son mari) ou «item con eundem legimus» (nous lisons chez le même auteur). Par la suite, l'hypothèse de Brøndal ne sera pas retenue. 
une nouvelle fois, actualiser les informations sémantiques véhiculées par le formant en question. L'existence de la relation $x R y \rightarrow y R x$ peut être vérifiée grâce à l'emploi de être (dont une des valeurs consiste à établir l'identité entre les référents des syntagmes nominaux que ce verbe met en correspondance, $\mathrm{cf}$. la notion de «equative sentences» chez Cann 1993: 31) comme dans les exemples 7.

7a. Jean est copain de Paul

7b. Paul est copain de Jean

7c. Jean et Paul sont copains

7d. Paul et Jean sont copains

Les prédicats monadiques qui ne sont pas, par définition, symétriques peuvent, eux aussi, à condition de leur ajouter un syntagme comitatif introduit par avec, acquérir un tel caractère. La différence entre ce type de verbes et ceux qui commencent par co- (décrits ci-dessus) consiste dans les modèles syntagmatiques qu'ils réalisent. Tandis que les premiers ne postulent qu'un argument, les verbes avec le formant $c o$-impliquent la présence d'au moins deux actants sémantiquement égaux. Les prédicats monadiques acquièrent donc, dans le meilleur des cas, le caractère symétrique secondairement et occasionnellement. A proprement parler, ils vont de pair avec le morphème grammatical (synsémantique) - avec (et la locution prépositionnelle en compagnie de) - pour actualiser cette propriété logique. C'est ainsi que dans $8 \mathrm{a}$ se promener est au centre de la prédication faite à propos d'un actant animé (et d'un autre, à caractère local, librement attaché au verbe). Il en est autrement dès qu'un syntagme comitatif (p. ex. avec Jacques) est inséré dans la phrase de départ. Comme résultat, on obtient la construction $8 \mathrm{~b}$ où, à l'opposé de $8 \mathrm{a}$, la relation entre les individus est symétrique.

\section{8a. Je me promène toujours aux alentours du parc municipal}

8 b. Je me promène toujours aux alentours du parc municipal avec Jacques

Du point de vue de sa valeur logique, la phrase $8 \mathrm{~b}$ est équivalente à Jacques se promène toujours aux alentours du parc municipal avec moi. L'unique différence est due au changement de la structure thème-propos. On voit donc quelle est l'influence du procédé syntaxique en question - il adjoint à l'expression prédicative un argument (d'ailleurs non postulé par la rection du verbe). Cette opération a pour conséquence la transformation des phrases où il n'y a pas de symétrie en constructions où une même propriété est attribuée à chacun des actants ${ }^{9}$. Le parcourfde cette transformation est représenté par 9.

${ }^{9}$ Ceci fait s'interroger sur l'utilité des descriptions qui consistent à appuyer l'interprétation sémantique d'une phrase sur l'organisation syntaxique de celle-ci. Cette idée a été prônée par lcs partisans de la grammaire générative (cf. Katz et Postal 1964: 20 «... the semantic interpretation of a sentence is under the empirical requirement to characterize the meaning of each and every constituent $[\ldots]$ of the sentence, and not to characterize the meaning for any substring of the sentence, that is not one of its constituents. [...] For example, the semantic interpretation of the sentence (9) the man hit the 
9.

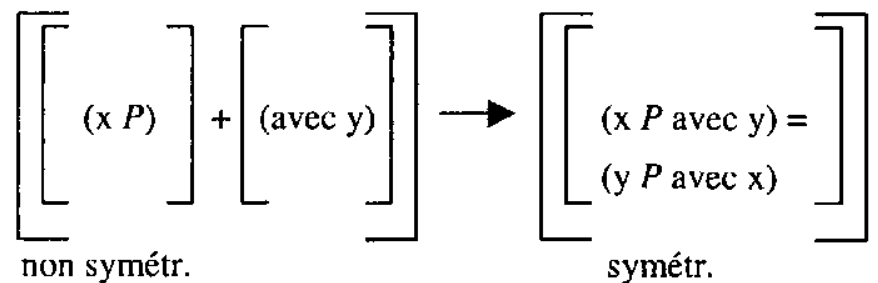

Il est pertinent de se poser la question sur l'universalité de cette transformation. La définition de la symétrie dans 1 stipule que les arguments mis en correspondance doivent appartenir à un même ensemble. En termes de la sémantique linguistique, ceci veut dire que les participants à la relation doivent être d'une même nature. Il devient, d'ores et déjà, clair quelles sont les limitations de la symétrie établie grâce à l'emploi de avec. Elles viennent du non respect de la contrainte sur l'homogénéité sémantique des arguments. C'est ainsi que les difficultés que pose, de ce point de vue, le couple $10 \mathrm{a}$ et $10 \mathrm{~b}$ sont liées à l'emploi, en guise d'arguments, des noms désignant les objets appartenant à des catégories ontologiques hétérogènes (en l'occurrence dotés de traits + humain et - humain).

10a. Les enfants se promènent avec la valise

10b. ? La valise se promène avec les enfants

Enfin, le dernier exposant de la symétrie qui sera mentionné ici est l'expression l'un l'autre (et ses variantes). La fonction primaire de celle-ci est analogue à celle du préfixe entre. Elle permet de lever l'ambiguïté inhérente aux constructions avec les verbes pronominaux. Comme il en a déjà été question, une phrase comme Pierre et Marie s'aiment reçoit tantôt une lecture réfléchie, tantôt réciproque. L'adjonction à cette phrase de l'un l'autre permet de conférer au verbe une interprétation symétrique. Cependant, le domaine d'emploi de l'expression en question est plus vaste que celui du préfixe entre-. Alors que ce dernier s'ajoute de préférence aux verbes transitifs, ce n'est pas le cas de l'un l'autre. Cette expression connaît des variantes d'emploi. C'est ainsi qu'auprès des verbes intransitifs il est nécessaire

ball must represent the meanings of the constituents of this sentence, i.e., the, man, hit, the, ball, the man, hit the ball, and the man hit the ball. But it must not provide any meaning for such substrings of (9) as the man hit or hit the»). Dans cette optique, linterprétation sémantique est secondaire par rapport à la description grammaticale. L'étude d'une phrase doit commencer par la syntaxc. Au moment où celle-ci est définitivement (en termes des constituants immédiats) établie, il est possible d'attribuer leurs sens aux nouds d'une image arborescente (qui rend compte de l'organisation syntaxique des phrases). L'analyse grammaticale est donc l'entréc de la sémantique. On voit, à l'excmple de l'amplification réalisée par avec, que le recours aux constituants immédiats est parfois insuffisant. Quand il en vient à la description du sens des phrases où il y a une relation symétrique, c'est plutôt le verbe suivi de la préposition qui cst le noyau prédicatif, bien qu'un tel amalgame ne soit pas constituant immédiat. Cf. toutefois le point de vue opposé chez Cervoni et sa discussion autour de Ce livre appartient, si je ne me trompe, au profe'sseur $\mathrm{ct} \mathrm{dc}{ }^{*}$ Ce livre appartient au, si je ne me trompe, professeur (1991: 110). 
d'insérer une préposition entre ses parties constitutives. Cette variation s'observe dans les exemples $11 \mathrm{a}$ et $11 \mathrm{~b}$. La parité de la prédication se traduit ici à travers la possibilité de permuter l'ordre des arguments. De même, si les deux actants sont homogènes, leurs noms peuvent être remplacés par une forme pronominale plurielle (voir 13). On voit donc que les caractéristiques syntaxiques des phrases contenant l'expression en question ne diffèrent pas de celles qui ont déjà été repérées.

11a. Ton mariage et mon départ ont fatalement coïncidé l'un avec l'autre

11b. Mon départ et ton mariage ont fatalement coincidé l'un avec l'autre

12. Les races les plus diverses se mêlent les unes aux autres dans la ville de Lyon

13. Elles se mêlent les unes aux autres dans la ville de Singapour

Il n'est pas rare de trouver des phrases où la présence de l'un l'autre a un caractère pléonastique. Une telle situation a lieu si cette expression suit un verbe symétrique (voir $11 \mathrm{a}$ et $11 \mathrm{~b}$ ). Il en est de même là où le verbe actualisant une notion symétrique est transitif (lls s'accompagnent les uns les autres). Dans tous ces exemples, l'insertion de l'un l'autre n'est pas requise pour signaler la symétrie (Ton mariage et mon départ ont fatalement coïncidé est suffisamment informatif de ce point de vue). Cependant, et c'est un autre trait distinctif de l'un l'autre, cette construction est rattachable également aux phrases où il n'y a pas de symétrie (voir Winter 2001: 339-345). Cf. les exemples 14.

14a. Les rois de cette dynastie se sont succédé les uns aux autres

14b. Dans sa chambre, les livres sont empilés les uns sur les autres

L'existence de ce type de phrases prouve que c'est le prédicateur (éventuellement suivi d'une préposition) qui est l'unique responsable de la présence de la symétrie. L'un l'autre est soit pléonastique (ce qui l'oppose à entre-, cf. la note $3 \mathrm{ci-}$ dessus), soit son rôle se limite à lever l'équivoque due à l'emploi du pronom personnel.

Les remarques qui suivent concernent la façon de rédiger les entrées dans les dictionnaires adressés au grand public. La productivité du préfixe entre- et la fréquence des verbes à préfixe co- dans le système lexical français ne sont pas passées inaperçues. Toutefois, ces formants sont souvent traités d'une façon peu précise. C'est ainsi qu'on retrouve dans Lexis (édition de 1993) les remarques suivantes à propos de la valeur de co-qui nous intéresse:

élément, du lat. cum, avec, entrant dans la composition de nombreux mots où il indique l'association, la participation, la simultanéité: coauteur, coexister, cohabiter, etc.

Il en est de même avec entre-, avec et l'un l'autre. Les informations qui s'y rapportent (et les emplois cités en guise d'illustration) sont insuffisantes, même pour les individus non concernés par les problèmes de la linguistique théorique. Les indications de ce type devraient, pour bien remplir leur tâche, mentionner les 
conséquences pratiques dues à la présence des formants en question dans la structure des unités lexicales. Ces «conséquences pratiques» concernent le comportement syntagmatique des termes placés dans les dictionnaires. Leurs modèles diathétiques, loin d'être fortuits, sont liés de près à la valeur qu'ont les parties constitutives de l'expression prédicative.

L'autre remarque est une récapitulation et concerne l'état quantitatif et qualitatif des exposants de la symétrie en français. Ceux-ci forment un système peu redondant, ce qui veut dire que les emplois et les valeurs des formants qui permettent d'actualiser la symétrie ne se recoupent généralement pas. Si tant est que la présence de co- soit motivée dans un dérivé synchronique, celui-ci est obligatoirement symétrique, ce qui se reconnaît à travers l'étude de son comportement syntaxique. Le statut de entre- est différent. Ce préfixe, tout en étant productif (les composés dont il fait partie sont pleinement motivés), connaît certaines restrictions quant à la nature de la base verbale à laquelle il est possible de l'attacher (avec les prédicats monadiques, il signifie «incomplètement, partiellement»). Sa fonction de base consiste à lever l'ambiguïté caractéristique des phrases avec un verbe pronominal (Marie et Pierre s'aiment). Avec, à son tour, sert à amplifier la valence des prédicats monadiques (étant donné que les dyadiques sont souvent causatifs, l'introduction de avec finit par ajouter à la phrase l'idée d'instrument) et à conférer à ceux-ci un caractère symétrique. Enfin, les emplois de l'un l'autre concurrencent partiellement ceux de entre-.

La redondance (c.à.d. la possibilité de prévoir la présence et la structure des segments qui suivent grâce à la connaissance de la nature sémantique de ceux qui précèdent) se produit là où: a) les verbes à préfixe $c o$-sont obligatoirement suivis d'un syntagme introduit par avec (redoublement des informations sur l'accompagnement); b) l'un avec l'autre succède aux verbes symétriques commençant par co-(Ces événements ont coïncidé l'un avec l'autre à l'opposé de Ces événements ont coïncidé où l'informativité de la construction est préservée même si le segment l'un l'autre est éliminé); 3) l'un l'autre est postposé au verbe réciproque commençant par entre- (cette expression est alors responsable de la réitération de la valeur imputable au préfixe en question).

\section{BIBLIOGRAPHIE}

Apresjan, J. (2000), Semantyka leksykalna. Synonimiczne srodki jezyka, Zaklad Narodowy im. Ossolińskich, Wroclaw-Warszawa-Kraków.

Aronoff, M. (1976), Word Formation in Generative Grammar, MIT Press, Cambridge, Massachusetts.

Banyś, W., Bednarczuk, L., Karolak, S. (eds.), Studia lingwistyczne ofiarowane Profesorowi Kazimierzowi Polańskiemu na 70-lecie Jego urodzin, Wydawnictwo Uniwersytetu Śląskiego, Katowice.

Bańczerowski, J. (1980), Systems of Semantics and Syntax. A determinational theory of language, PWN, Warszawa-Poznań. 
Brøndal, V. (1950), Théorie des prépositions. Introduction à une sémantique rationnelle, Ejnar Munksgaard, Copenhague.

Can n, R. (1993), Formal Semantics. An Introduction, Cambridge University Press, Cambridge.

Cervoni, J. (1991), La préposition. Etude sémantique et pragmatique, Duculot, Paris-Louvain-la Neuve.

Cresswell, M. (1973), Logics and Languages, Methuen \& Co Ltd., London.

Fillmore, C. J. (1968), Case for Case, in: E. Bach, R.T. Harms (eds.), Universals in linguistic Theory, Holt, Rinehart \& Winston, Inc., New York, pp. 1-88.

Fouché, P. (1961), Phonétique historique du français, vol. 3, Kilncksieck, Paris.

Giermak-Zielińska, T. (1979), Polskie czasowniki przedrostkowe o znaczeniu przestrzennym i ich odpowiedniki w jezyku francuskim, Zakład Narodowy im. Ossolińskich, Wroclaw-WarszawaKraków-Gdańsk.

Grzcgorczykowa, R., Puzynina, J. (1999), Slowotwórstwo, in: R. Grzegorczykowa, R. Laskowski, H. Wróbel, (red.), Gramatyka współczesnego języka polskiego. Morfologia, vol. 2, Wydawnictwo Naukowe PWN, Warszawa, pp. 361-388.

I mbs, P. (1956), Les propositions temporelles en ancien français. La détermination du moment, Les Belles Lettres, Paris.

Karolak, S. (1990), Kwantyfikacja a determinacja w jezykach naturalnych, PWN, Warszawa.

Karolak, S. (1999), O pojęciu strukturalnego schematu zdania, in: W. Banys, L. Bednarczuk, S. Karolak, (red.), pp. 129-137.

Katz, J.J., Postal, P.M. (1964), An Integrated Theory of Linguistic Description, MIT Press, Cambridge, Massachusetts.

Kempf, Z. (1978), Próba teorii przypadków, Opolskie Towarzystwo Przyjaciół Nauk - Wydział Języka i Literatury, Wrocław.

Korytkowska, M. (1999), Diateza a predykatory analityczne $w$ jezyku polskim i bufgarskim, in: W. Banyś, L. Bednarczuk, S. Karolak, (eds.), pp. 138-145.

La miroy, B. (1993), L'incomplétude du passif dans les langues romanes, in: S. Karolak, T. Muryn, (eds.), Complétude et incomplétude dans les langues romanes et slaves. Actes du Vl colloque international de linguistique romane et slave. Cracovie 29 septembre - 3 octobre 1991, Ecole Normale Supéricure - Institut d'Etudes Romanes, Krakow, pp. 241-266.

Lewicka, H., B ogacki, K. (eds.), (1983), Dictionnaire sémantique et syntaxique des verbes français, PWN, Warszawa.

Lexis. Dictionnaire de la langue française (1993), Larousse, Paris.

Matthews, P.H. (1991), Morphology, Cambridge University Press, Cambridge.

Nagórko, A. (2001), Zarys gramatyki polskiej (ze slowotwórstwem), Wydawnictwa Naukowe PWN, Warszawa.

Winter, Y. (2001), Plural Predication and the Strongest Meaning Ilypothesis, in: Journal of Semantics 18 (4), pp. 333-365. 